\title{
Towards Average-Case Complexity Analysis of NP Optimization Problems*
}

\author{
Rainer SCHULER \\ Abteilung Theoretische Informatik \\ Universität Ulm \\ Oberer Eselsberg \\ D 89069 Ulm, Germany \\ schuler@informatik.uni-ulm.de
}

\author{
Osamu WATANABE \\ Department of Computer Science \\ Tokyo Institute of Technology \\ Meguro-ku Ookayama 1-12-1 \\ Tokyo 152, JAPAN \\ watanabe@cs.titech.ac.jp
}

\begin{abstract}
For the worst-case complexity measure, if $\mathrm{P}=\mathrm{NP}$, then $\mathrm{P}=\mathrm{OptP}$, i.e., all NP optimization problems are polynomial-time solvable. On the other hand, it is not clear whether a similar relation holds when considering average-case complexity. We investigate the relationship between the complexity of NP decision problems and that of NP optimization problems under polynomial-time computable distributions, and study what makes them (seemingly) different. It is shown that the difference between $\mathrm{P}_{\mathrm{tt}}^{\mathrm{NP}}$-samplable and $\mathrm{P}^{\mathrm{NP}}$-samplable distributions is crucial.
\end{abstract}

\section{Introduction}

Recently, "average-case complexity" has received considerable attention by researchers in several fields of computer science. Even a problem is not (or may not be) solvable efficiently in the worst-case, it may be solvable efficiently on average. Indeed, several results have been obtained that show even simple algorithms work well on average (see, e.g., [Joh84]). On the other hand, most of those results are about concrete problems, and not so much has been done for more general study of average-case complexity, though there are many interesting open questions in this area. In this paper, we consider one of such open questions, and improve our knowledge towards this question.

We consider the following question: Suppose every NP problem is polynomial time

\footnotetext{
*The part of this work has been done while the second author was visiting Universität Ulm and supported in part by the guest scientific program of Universität Ulm. The second author is supported in part by Grant in Aid for Scientific Research of the Ministry of Education, Science and Culture of Japan under Grant-in-Aid for Research (C) 06680308 (1994).
} 
solvable on average. Does this mean that every NP optimization problem is also polynomial time solvable on average? Here "NP problem" is a decision problem for an NP set. On the other hand, "NP optimization problem" is a problem of finding optimal solutions for a problem with a polynomial-time computable cost function. Krentel [Kre88] defined the class OptP for the class of NP optimization problems. Thus, the question is whether $\mathrm{P}=\mathrm{NP}$ on average implies $\mathrm{P}=\mathrm{OptP}$ on average. (Since OptP is the class of functions, "P $=\mathrm{OptP}$ " should be written as "PF $=\mathrm{OptP}$ ". In this paper, however, we will use $\mathrm{P}$ to denote both language and function classes.)

For discussing average-case complexity, one should be careful about input distributions and distribution classes. It may not be so realistic to discuss polynomial-time computability considering any input distribution. Levin [Lev86], who established a framework for average-case complexity theory, proposed to consider only "polynomial-time computable distribution (in short, P-computable distribution)" as input distributions. Later more generalized notion, i.e., "polynomial-time samplable distribution (in short, P-samplable distribution)", has been proposed [BCGL92]. We essentially follow Levin's framework, and regard P-computable distributions (or P-samplable distributions) as realistic input distributions. Thus, by "P $=$ NP on average" we mean that for every NP problem and every P-computable distribution, the problem is solvable in polynomialtime on average when an input instance is given under the distribution. (In this introduction, we will use, e.g., "P = ave $\mathrm{NP}$ (under P-comp. dist.)" to mean "P $=\mathrm{NP}$ on average for any P-computable distribution.")

For the worst-case complexity measure, we have $\mathrm{P}=\mathrm{NP} \Longrightarrow \mathrm{P}=\mathrm{OptP}$. This is from the following reason: Every NP optimization problem $A$ is polynomial-time solvable by some algorithm $Q$ by using some $\mathrm{NP}$ set $X$ as an oracle. But since $\mathrm{P}=$ NP, we can replace oracle $X$ with some polynomial-time machine $M$ for $X$. Thus, $Q^{M}$ solves $X$ in polynomial-time. This simple argument does not work, however, in the average-case complexity. Even if $X$ is solvable by $M$ in polynomial-time on average under any P-computable distribution, this does not mean that $Q^{M}$ runs in polynomialtime on average under every $\mathrm{P}$-computable distribution. This is because queries to $X$ may occur under a very strange distribution, for which no algorithm solves $X$ in polynomial-time on average. Thus, it is not clear that the relation $\mathrm{P}={ }_{\text {ave }} \mathrm{NP}$ (under P-comp. dist.) $\Longrightarrow \mathrm{P}=$ ave OptP (under P-comp. dist.) holds; or, it may not hold at all. In this paper, we study what makes this relation difficult.

We consider two approaches. First, we investigate how much we need to enlarge a distribution class $\mathcal{D}_{1}$ so that the following implication holds: $\mathrm{P}=$ ave $\mathrm{NP}$ (under $\mathcal{D}_{1}$ dist.) $\Longrightarrow \mathrm{P}=$ ave OptP (under P-comp. dist.). Secondly, we consider for which class $\mathcal{D}_{2}$ 
can we prove the following implication: $\mathrm{P}={ }_{\text {ave }} \mathrm{NP}$ (under $\mathrm{P}$-comp. dist.) $\Longrightarrow \mathrm{P}=$ ave NP (under $\mathcal{D}_{2}$ dist.). Obviously, if $\mathcal{D}_{1} \subseteq \mathcal{D}_{2}$, then we have an affirmative answer to our question. While we have been unable to achieve this, we can prove the following results.

(1) If $\mathrm{P}={ }_{\text {ave }} \mathrm{NP}$ under every $\mathrm{P}^{\mathrm{NP}}$-samplable distribution, then $\mathrm{P}={ }_{\text {ave }}$ OptP under every P-computable distribution. Furthermore, the converse relation holds. That is, the assumption is indeed necessary for showing $\mathrm{P}={ }_{\text {ave }}$ OptP (under $\mathrm{P}$-comp. dist.).

(2) If $\mathrm{P}={ }_{\text {ave }} \mathrm{NP}$ under every P-computable distribution, then $\mathrm{P}={ }_{\text {ave }} \mathrm{NP}$ under every $\mathrm{P}_{\mathrm{tt}}^{\mathrm{NP}}$-samplable distribution.

Thus, we now know that the difference between $\mathrm{P}_{\mathrm{tt}}^{\mathrm{NP}}$-samplable and $\mathrm{P}^{\mathrm{NP}}$-samplable distributions is crucial for our question. Motivated by this, we also study how strong $\mathrm{P}_{\mathrm{tt}}^{\mathrm{NP}}$-samplable distributions are, and obtain the following result.

(3) Every \#P-computable distribution can be approximated within constant factor by some $\mathrm{P}_{\mathrm{tt}}^{\mathrm{NP}}$-samplable distribution.

Thus, from this and the above result (2), we can show that \#P-computable distributions are not stronger than P-computable distributions for discussing the average-case polynomial-time computability of NP.

Impagliazzo and Levin [IL90] made an important observation on different classes of distributions. They showed that if $\mathrm{P}={ }_{\text {ave }} \mathrm{NP}$ (under P-comp. dist.), then it indeed holds that $\mathrm{P}={ }_{\text {ave }} \mathrm{NP}$ (under $\mathrm{P}$-samplable dist.). For obtaining the above results (1) and (2), we extend their technique and prove that if $\mathrm{P}={ }_{\text {ave }} \mathrm{NP}$ (under P-comp. dist.), then $\mathrm{P}=$ ave NP even for any "average" P-samplable distribution. For showing (3), we use another property of hash functions.

\section{Preliminaries}

In this paper, we follow the standard definitions and notations in computational complexity theory (see, e.g., [BDG88, BDG91]).

Throughout this paper, we fix our alphabet to $\Sigma=\{0,1\}$, and by a string we mean an element of $\Sigma^{*}$. For any string $x$, let $|x|$ denote the length of $x$. For any $n \geq 0$ and any set $L$ of strings, let $L^{\leq n}$ and $L^{=n}$ be the set of strings in $L$ of length $\leq n$ and of length $n$ respectively. We use $\|L\|$ to denote the cardinality of $L$. Let $\mathrm{N}$ denote the set of nonnegative integers. Usually, we assume the binary encoding of $\mathbf{N}$ on $\Sigma^{*}$, but sometimes numbers are encoded in a tally form, i.e., as a string in $0^{*}$. For any $n \in \mathbf{N}$, let $\bar{n}$ denote $0^{n}$. 
We use a standard one-to-one pairing function from $\Sigma^{*} \times \Sigma^{*}$ to $\Sigma^{*}$ that is computable and invertible in polynomial-time. For inputs $x$ and $y$, we denote the output of the pairing function by $\langle x, y\rangle$; this notation is extended to denote any $n$ tuple. We also use a polynomial-time computable pairing function, say, $\langle n, x, y\rangle_{n}$ such that for every $n \geq 0$ and for all $(x, y)$ in some finite set $D_{n},\langle n, x, y\rangle_{n}$ is of the same length, which is uniquely determined by $n$. (We assume that $\langle n, x, y\rangle_{n}$ is undefined for $(x, y) \notin D_{n}$.) It is not so hard to define such pairing functions by using standard padding technique. We often omit specifying $D_{n}$ when it is clear from the context.

For any random event $\alpha$, let $\operatorname{Pr}_{\alpha}\{\Phi(\alpha)\}$ be the probability $\Phi(\alpha)$ holds. For example, for any randomized machine $M, \operatorname{Pr}_{M}\{\Phi\}$ is the probability that $\Phi$ holds when $M$ executes following its internal coin tosses; $\operatorname{Pr}_{x \in U}\{\Phi(x)\}$ is the probability that $\Phi$ holds when $x$ is chosen from $U$ randomly. The latter one is also written as $\operatorname{Pr}\{\Phi(x) \mid x \in U\}$.

For our computation model, we use randomized (oracle) Turing machines. We say that a machine $M$ accepts a language $L$ if for all $x \in \Sigma^{*}$,

$$
\begin{aligned}
& x \in L \Longrightarrow \operatorname{Pr}_{M}\{M \text { accepts } x\} \geq 2 / 3, \text { and } \\
& x \notin L \Longrightarrow \operatorname{Pr}_{M}\{M \text { accepts } x\} \leq 1 / 3 .
\end{aligned}
$$

Similarly, for any (multi-valued) function $f$, we say that $f$ is computed by $M$ if for every $x$, the probability that $M(x)$ computes $f(x)$ is greater than $2 / 3$. Note that for any decision problem and any single-valued function evaluation problem, we can easily reduce the error probability by executing a machine several times and then taking their majority. Also even for evaluating a multi-valued function, if it is easy to verify the correctness of a given answer, then we can easily reduce the error probability.

Throughout this paper, we use this randomized polynomial-time computability instead of the deterministic one. Thus, by " $f$ is polynomial-time computable", we precisely mean that $f$ is polynomial-time computable by some randomized machine. Note that for a randomized machine $M$ and any input $x$, the running time of $M$ on $x$ may differ depending on the random sequence that $M$ uses. The running time of $M$ on $x$ (written as time ${ }_{M}(x)$ ) is formally defined as an expected running time of $M$ on $x$ over all random sequences of $M$.

Optimization problems we consider are specified by polynomial-time computable functions. For a polynomial-time computable function cost: $\Sigma^{*} \times \Sigma^{*} \rightarrow \mathrm{N}$, and a polynomial $p$, the NP optimization problem specified by cost and $p$ is to compute the following function opt-val:

$$
\operatorname{opt}-\operatorname{val}(x)=y \in \Sigma^{\leq p(|x|)} \text { such that } \operatorname{cost}(x, y)=\operatorname{opt}(x) \text {, }
$$


where $\operatorname{opt}(x)=\max \left\{\operatorname{cost}\left(x, y^{\prime}\right) \mid y^{\prime} \in \Sigma^{\leq p(|x|)}\right\}$. Notice that opt-val is multi-valued in general. OptP is the class of functions like opt-val. (In order to keep similarity with "NP search problem", we modified the original definition of OptP [Kre88], where OptP is defined as the class of functions like opt. It is, however, easy to show that the above class OptP is a generalization of the original class, and they are essentially the same for discussing polynomial-time computability.)

For a given NP set $L$, an NP search problem for $L$ is to search, for a given instance $x$ in $L$, some witness for $x \in L$. More formally, for a polynomial-time computable predicate $R$ on $\Sigma^{*} \times \Sigma^{*}$, and a polynomial $p$, the NP search problem specified by $R$ and $p$ is to compute a value of the following function search:

$$
\operatorname{search}(x)=y \in \Sigma^{\leq p(|x|)} \text { such that } R(x, y) \text { holds. }
$$

Notice again that search may be multi-valued in general. Let SearchP denote the class of functions like search.

Preliminaries for Average-Case Complexity Theory

A probability function $\mu$ on $U$ is a total function from $U$ to $[0,1]$ such that $\sum_{x \in U} \mu(x)$ $=1$. We use $\lambda$ to denote the uniform probability function on $\Sigma^{r}$, where $r$ will be specified in each context.

Throughout this paper, only length-wise input distributions are considered. That is, for each $n \geq 0$, we consider probability function $\mu_{n}$ on $\Sigma^{n}$ (hence, $\sum_{x \in \Sigma^{n}} \mu_{n}(x)=1$ ), and discuss average-case complexity assuming that each instance $x \in \Sigma^{n}$ appears with probability $\mu_{n}(x)$. Thus, formally speaking, an input distribution (or, distribution in short) is specified as a family $\left\{\mu_{n}\right\}_{n \geq 0}$ of such length-wise probability functions $\mu_{n}$. In this paper, however, we denote an input distribution by a single function such as $\mu$, and for each $x \in \Sigma^{n}$, we use $\mu(x)$ to denote $\mu_{n}(x)$.

Definition 2.1. ( $\mathrm{P}^{\mathcal{C}}$-computable distribution, $\mathrm{P}_{\mathrm{tt}}^{\mathcal{C}}$-computable distribution) For any complexity class $\mathcal{C}$ and any input distribution $\mu, \mu$ is a $P^{\mathcal{C}}$-computable distribution if its cumulative distribution $\mu^{*}$ is computed by some polynomial-time bounded randomized oracle Turing machine $M$ relative to some oracle set $X \in \mathcal{C}$. The notion of " $\mathrm{P}_{\mathrm{tt}}^{\mathcal{C}}$-computable distribution" is defined similarly by considering oracle Turing machines that ask queries only nonadaptively.

Remark. We are using the randomized polynomial-time computability for the "polynomial-time computability" notion. Furthermore, we are using length-wise probability, and the cumulative distribution $\mu^{*}$ of $\mu$ is defined by $\mu^{*}(x)=\sum_{x^{\prime}: x^{\prime} \in \Sigma^{n} \wedge x^{\prime} \leq x} \mu\left(x^{\prime}\right)$, where $\leq$ is the standard lexicographic order on $\Sigma^{*}$. Thus, the above definition is not 
equivalent to the original one in [Lev86]. Nevertheless, the following argument does not change even if the above notion is defined by using the deterministic polynomial-time computability. In this case, it is easy to show that our definition is equivalent to the original one as long as the probability for each length is polynomial-time computable.

Definition 2.2. ( $\mathrm{P}^{\mathcal{C}}$-samplable distribution, $\mathrm{P}_{\mathrm{tt}}^{\mathcal{C}}$-samplable distribution) For any complexity class $\mathcal{C}$ and any input distribution $\mu, \mu$ is a $P^{\mathcal{C}}$-samplable distribution if there exist a polynomial-time bounded randomized oracle Turing machine $G$, which is called a generator, and some set $X \in \mathcal{C}$ such that for each $x$ (let $n=|x|$ ),

$$
\mu(x)=\operatorname{Pr}_{G}\left\{G^{X}(\bar{n}) \text { yields } x\right\}
$$

The notion of " $\mathrm{P}_{\mathrm{tt}}^{\mathcal{C}}$-samplable distribution" is defined similarly by considering oracle Turing machines that ask queries only nonadaptively.

We define distribution classes. For any complexity class $\mathcal{C}$, let $\mathrm{P}^{\mathcal{C}}$-comp denote the classes of distributions that are $\mathrm{P}^{\mathcal{C}}$-computable. Let $\mathrm{P}^{\mathcal{C}}$-samp (resp., $\mathrm{P}_{\mathrm{tt}}^{\mathcal{C}}$-samp) denote the class of $\mathrm{P}^{\mathcal{C}}$-samplable (resp., $\mathrm{P}_{\mathrm{tt}}^{\mathcal{C}}$-samplable) distributions. Though we defined notions in a general way, we will mainly consider distribution classes P-comp, P-samp, $\mathrm{P}_{\mathrm{tt}}^{\mathrm{NP}}$-samp, and $\mathrm{P}^{\mathrm{NP}}$-samp.

Note that by these definitions, the values of probability functions are always (binary) rational numbers. Thus, these definitions are weaker than the original ones [Lev86, BCGL92] that allow real numbers for probability. Nevertheless, it is shown [Gur91, Lemma 1.6] that we lose no generality by this restriction for discussing polynomial-time computability.

Levin [Lev86] gave a general and robust definition to the notion of "polynomial-time solvable on average". Levin's definition uses distributions on $\Sigma^{*}$; on the other hand, we are using length-wise input distributions in order to make our discussion more intuitive. Thus, we modify Levin's definition to a length-wise version, which is more intuitive but less robust. The following definition for "polynomial on $\mu$-average" was suggested by Gurevich [Gur91].

Definition 2.3. (Polynomial on $\mu$-average)

A function $f$ is polynomial on $\mu$-average if there exist constants $c, d>0$ such that for all $n \geq 0$,

$$
\sum_{x \in \Sigma^{n}} \frac{f(x)^{1 / d}}{n} \mu(x) \leq c .
$$


For showing "polynomial on $\mu$-average", the following simple characterizations are useful.

Proposition 2.4. Let $f$ be any function from $\Sigma^{*}$ to $\mathbf{N}$, and $\mu$ be any input distribution. Then $f$ is polynomial on $\mu$-average if there exist polynomials $p$ and $q$ and a constant $d>0$ that satisfy the following for all $n \geq 0$ :

$$
\sum_{x \in \Sigma^{n}} \frac{f(x)^{1 / d}}{q(n)} \mu(x) \leq p(n)
$$

Remark. The proposition is provable by using an argument similar to the proof of [Gur91, Lemma 1.5].

Proposition 2.5. Let $f$ and $t$ be any functions from $\Sigma^{*}$ to $\mathbf{N}$ and from $\mathbf{N}$ to $\mathbf{N}$ respectively, and let $\mu$ be any input distribution. For any $k \geq 1$, assume that $f$ is polynomial in $t(n)^{k}$ on $\mu$-average; that is, for some constants $c, d>0$ and for all $n \geq 0$, we have

$$
\sum_{x \in \Sigma^{n}} \frac{f(x)^{1 / d}}{t(n)^{k}} \mu(x) \leq c .
$$

Then for any set $X \subseteq \Sigma^{*}$ such that $\mu\left(X \cap \Sigma^{n}\right) \leq 1 / t(n)$ for all $n \geq 0, f$ is polynomial on $\mu$-average on $X$; or more specifically, for all $n \geq 0$ and $X^{\prime} \subseteq \Sigma^{n}$ such that $\mu\left(X^{\prime}\right) \leq$ $1 / t(n)$, we have

$$
\sum_{x \in X^{\prime}} f(x)^{1 /(2 d k)} \cdot \mu(x) \leq c+1
$$

Proof. We split the sum depending on the value of $f(x)^{1 /(2 d k)}$.

(1) Let $S$ be the set of all $x \in X^{\prime}$ with $f(x)^{1 /(2 d k)} \leq t(n)$. Then

$$
\sum_{x \in S} f(x)^{1 /(2 d k)} \cdot \mu(x) \leq \sum_{x \in S} t(n) \cdot \mu(x)=t(n) \cdot \sum_{x \in S} \mu(x) \leq t(n) \cdot \frac{1}{t(n)}=1 .
$$

(2) Let $T$ be the set of all $x \in X^{\prime}$ with $f(x)^{1 /(2 d k)}>t(n)$, i.e $f(x)^{1 /(2 d)}>t(n)^{k}$. Then

$$
\begin{aligned}
& \sum_{x \in T} f(x)^{1 /(2 d k)} \cdot \mu(x)=\sum_{x \in T} \frac{t(n)^{k} \cdot f(x)^{1 /(2 d k)}}{t(n)^{k}} \mu(x) \\
& \leq \sum_{x \in T} \frac{f(x)^{1 /(2 d)} \cdot f(x)^{1 /(2 d k)}}{t(n)^{k}} \mu(x) \leq \sum_{x \in T} \frac{f(x)^{1 / d}}{t(n)^{k}} \mu(x) \leq c .
\end{aligned}
$$

We say that a machine $M$ runs in polynomial-time on $\mu$-average if its (expected) running time time $M$ is polynomial on $\mu$-average. It is shown [Gur91, Proposition 1.1] that the above definition is equivalent to Levin's original one for distributions satisfying a 
certain natural condition. Furthermore, all the arguments in this paper can be modified for Levin's definition. Thus, we will lose no generality by using this definition.

For our notion of "average polynomial-time", we will use the above definition in this paper. Nevertheless, we should also note that there are weaker (but still natural and robust) ways to define this notion. The following is one such example, which has been used in the study of cryptographic one-way functions.

Definition 2.6. (Almost polynomial under $\mu$ )

A function $f$ is almost polynomial under $\mu$ if there exist integer $k$ such that for all $c \geq 0$ and for almost all $n \geq 0$,

$$
\mu\left\{x \in \Sigma^{n} \mid f(x) \leq n^{k}\right\} \geq 1-\frac{1}{n^{c}}
$$

The reducibility notion often helps us to discuss the implication of complexity assumptions such as $\mathrm{P}=$ NP. Here we use the following reducibility from [BCGL92].

Definition 2.7. (Random reduction)

Let $\left(L_{1}, \mu_{1}\right)$ and $\left(L_{2}, \mu_{2}\right)$ be respectively a pair of a language and a probability function. A random reduction (or, more specifically, $\alpha_{\mathrm{T}}^{\mathrm{P}}$-reduction) from $\left(L_{1}, \mu_{1}\right)$ to $\left(L_{2}, \mu_{2}\right)$ is a randomized oracle Turing machine $Q$ with the following properties:

(a) $Q$ is polynomial-time bounded.

(b) For every $x \in \Sigma^{*}$,

$$
\begin{aligned}
& x \in L_{1} \Longrightarrow \operatorname{Pr}_{Q}\left\{Q^{L_{2}} \text { accepts } x\right\} \geq 2 / 3, \text { and } \\
& x \notin L_{1} \Longrightarrow \operatorname{Pr}_{Q}\left\{Q^{L_{2}} \text { rejects } x\right\} \geq 2 / 3 .
\end{aligned}
$$

(c) There exists a polynomial $p$ such that for any $n \geq 0$ and for every $y \in \Sigma^{*}$ that is queried by $Q^{L_{2}}$ on some $x \in \Sigma^{n}$, we have

$$
\mu_{2}(y) \geq \frac{1}{p(n)} \sum_{x \in \Sigma^{n}} \operatorname{Ask}_{Q}\left(x, y ; L_{2}\right) \cdot \mu_{1}(x),
$$

where $\operatorname{Ask}_{Q}\left(x, y ; L_{2}\right)$ is the probability $\operatorname{Pr}_{M}\left\{Q^{L_{2}}(x)\right.$ queries $\left.y\right\}$.

Remark. Here we modified the original definition for our length-wise probability functions. Some of the above conditions are slightly more restrictive than the original ones.

$\mathrm{A} \propto_{\mathrm{T}}^{\mathrm{P}}$-reduction $Q$ that asks queries only nonadaptively is called a $\propto_{\mathrm{tt}}^{\mathrm{P}}$-reduction. A $\propto_{\mathrm{T}}^{\mathrm{P}}$-reduction is extensively used from a (multi-valued) function to a set. It is easy to show that the following relation holds [BCGL92]. 
Proposition 2.8. Let $\left(L_{1}, \mu_{1}\right)$ and $\left(L_{2}, \mu_{2}\right)$ be respectively a pair of a language and a probability function. If $\left(L_{1}, \mu_{1}\right)$ is $\propto_{\mathrm{T}}^{\mathrm{P}}$-reducible to $\left(L_{2}, \mu_{2}\right)$, and $L_{2}$ is polynomial-time solvable on $\mu_{2}$-average, then $L_{1}$ is polynomial-time solvable on $\mu_{1}$-average.

Remark. This can be extended for a reduction from $\left(f_{1}, \mu_{1}\right)$ to $\left(L_{2}, \mu_{2}\right)$, for a (possibly multi-valued) function $f_{1}$.

\section{Known Results and Some Simple Observations}

For simplifying our statements, we will use the following complexity class introduced in [SY92].

Definition 3.1. (Class $\mathrm{P}_{\mathcal{D}}$ )

For any distribution class $\mathcal{D}, \mathrm{P}_{\mathcal{D}}$ is the class of languages $L$ such that for any distribution $\mu \in \mathcal{D}, L$ is accepted by some randomized Turing machine whose running time is polynomial on $\mu$-average.

Remark. We will also use this notation for discussing the complexity of computing functions. That is, a function $f$ is in $\mathrm{P}_{\mathcal{D}}$ if for any distribution $\mu \in \mathcal{D}, f$ is computable by some randomized Turing transducer whose running time is polynomial on $\mu$-average.

For example, $\mathrm{P}_{\mathrm{P}-\mathrm{comp}}$ is the class of languages that are polynomial-time solvable on average under any polynomial-time computable distributions. Hence, the relation NP $\subseteq \mathrm{P}_{\mathrm{P} \text {-comp }}$ is equivalent to the following statement: for all $\mathrm{P}$-computable distribution $\mu$ and all $L$ in NP, $L$ is polynomial-time decidable on $\mu$-average. Thus, by this notation, we can state our principal question as follows: Is it true that $\mathrm{NP} \subseteq \mathrm{P}_{\mathrm{P}-\text { comp }} \Longrightarrow \mathrm{OptP} \subseteq$ $\mathrm{P}_{\mathrm{P}-\text { comp }}$ ? Also two questions we asked in the introduction are stated as follows.

Q1. For which distribution class $\mathcal{D}$, do we have the following implication?:

$$
\mathrm{NP} \subseteq \mathrm{P}_{\mathcal{D}} \Longrightarrow \mathrm{OptP} \subseteq \mathrm{P}_{\mathrm{P}-\text { comp }}
$$

Q2. For which distribution class $\mathcal{D}$, do we have the following implication?:

$$
\mathrm{NP} \subseteq \mathrm{P}_{\mathrm{P}-\text { comp }} \Longrightarrow \mathrm{NP} \subseteq \mathrm{P}_{\mathcal{D}}
$$

Here let us review previous results.

Proposition 3.2. [BCGL92]

(1) $\mathrm{NP} \subseteq \mathrm{P}_{\mathrm{P} \text {-samp }} \Longrightarrow \Theta_{2}^{\mathrm{P}} \subseteq \mathrm{P}_{\mathrm{P} \text {-samp }}$ (where $\Theta_{2}^{\mathrm{P}}=\mathrm{P}_{\mathrm{tt}}^{\mathrm{NP}}$ ).

(2) $\mathrm{NP} \subseteq \mathrm{P}_{\mathrm{P} \text {-samp }} \Longrightarrow$ SearchP $\subseteq \mathrm{P}_{\mathrm{P} \text {-samp }}$.

Remark. The first fact, which is almost immediate from Proposition 2.8, is not explicitly stated in [BCGL92], but the idea has been used to show the second fact. 
Proposition 3.3. [IL90] $\mathrm{NP} \subseteq \mathrm{P}_{\mathrm{P}-\mathrm{comp}} \Longrightarrow \mathrm{NP} \subseteq \mathrm{P}_{\mathrm{P}-\mathrm{samp}}$.

By using these propositions, it is not so hard to show that if $\mathrm{NP} \subseteq \mathrm{P}_{\mathrm{P} \text {-comp }}$, then every NP optimization problem has an average-polynomial-time approximation scheme.

Consider any NP optimization problem $\Pi$, and let cost and $p$ be a cost function and a polynomial specifying $\Pi$. For any probability function $\mu$, we say that $\Pi$ has a $\mu$-average-polynomial-time approximation scheme if for each $\varepsilon, 0<\varepsilon<1$, there exists a randomized Turing machine $M$ with the following properties:

(a) The running time of $M$ is polynomial on $\mu$-average.

(b) For any $x \in \Sigma^{*}$, with probability greater than $2 / 3, M(x)$ yields an $\varepsilon$-approximation $y$; that is, $y$ satisfies

$$
\frac{\operatorname{opt}(x)-\operatorname{cost}(x, y)}{\operatorname{opt}(x)} \leq \varepsilon .
$$

(Recall that we assumed that $\operatorname{cost}(x, y) \geq 0$ and that we are considering maximization problems.)

Theorem 3.4. If $\mathrm{NP} \subseteq \mathrm{P}_{\mathrm{P}-\text { comp }}$, then every $\mathrm{NP}$ optimization problem has a $\mu$-averagepolynomial-time approximation scheme for every $\mathrm{P}$-computable distribution $\mu$.

Proof. (The same idea has been used for proving [CG93, Lemma 4].)

Let $\Pi$ be any NP optimization problem that is specified by cost and $p$. Also let $\mu$ be any P-computable distribution.

Consider any constant $\varepsilon, 0<\varepsilon<1$, and let it be fixed. We show some machine exists that satisfies the above conditions (a) and (b) for $\varepsilon$. Formally, the problem is to compute a (multi-valued) function $a p$-val whose value on $x$ takes every $\varepsilon$-approximation of $x$. We reduce this approximation problem to some NP search problem. Let opt $(x)$ $=\max \left\{\operatorname{cost}\left(x, y^{\prime}\right) \mid y^{\prime} \in \Sigma^{\leq p(|x|)}\right\}$. We may assume that for every $x \in \Sigma^{*}, 0 \leq \operatorname{opt}(x) \leq$ $2^{q(|x|)}$ for some polynomial $q$. Also let $r$ be a polynomial such that $\left\lfloor(1-\varepsilon)^{r(n)} 2^{q(n)}\right\rfloor=0$ for any $n$. Now we consider the problem of computing the following function:

For each $\langle n, x, k\rangle_{n}$, where $x \in \Sigma^{n}$ and $1 \leq k \leq r(n)$, $\operatorname{search}\left(\langle n, x, k\rangle_{n}\right)=y$ such that $\left\lfloor(1-\varepsilon)^{k} 2^{q(n)}\right\rfloor \leq \operatorname{cost}(x, y) \leq\left\lceil(1-\varepsilon)^{k-1} 2^{q(n)}\right\rceil$.

Clearly, if there is no solution for $\langle n, x, k-1\rangle_{n}$, then any solution for $\langle n, x, k\rangle_{n}$ is an $\varepsilon$-approximation. Thus, the problem of computing ap-vall $(x)$ is solvable by computing search for all $\langle n, x, k\rangle_{n}, 1 \leq k \leq r(n)$. That is, ap-val is polynomial-time reducible to search. Now it suffices to show some $\mu^{\prime}$ in $\mathrm{P}$-comp such that $(a p-v a l, \mu)$ is $\alpha_{\mathrm{T}}^{\mathrm{P}}$-reducible (in fact, $\alpha_{\mathrm{tt}}^{\mathrm{P}}$-reducible) to $\left(\right.$ search,$\left.\mu^{\prime}\right)$. This can be done by simply defining $\mu^{\prime}\left(\langle n, x, k\rangle_{n}\right)$ $=\mu(x) / r(|x|)$. 


\section{The First Question}

Here we discuss our first question. Namely, we would like to obtain some sufficient condition (in terms of the relation $\mathrm{NP} \subseteq \mathrm{P}_{\mathcal{D}}$ ) for all $\mathrm{NP}$ optimization problems to be polynomial-time solvable on average under any $\mathrm{P}$-computable distribution. For example, it has been shown [SY92] that $\mathrm{P}_{\mathrm{E}-\text { comp }}$ is exactly the same as $\mathrm{P}$, where E-comp is the class of exponential-time computable distributions. Hence, $\mathrm{NP} \subseteq \mathrm{P}_{\mathrm{E}-\text { comp }}$ implies that $\mathrm{P}$ $=\mathrm{NP}$ even in the worst-case; thus clearly, all NP optimization problems are polynomialtime solvable (even in the worst-case). Here we will show that a much weaker condition $\mathrm{NP} \subseteq \mathrm{P}_{\mathrm{P}^{N P} \text {-samp }}$ is sufficient, and furthermore it is indeed necessary.

Before proving this result, let us first show that we lose no generality if we discuss our problem by using $\Delta_{2}^{\mathrm{P}}$ class.

Theorem 4.1. For any distribution class $\mathcal{D}$ defined in Section 2, we have $\Delta_{2}^{\mathrm{P}} \subseteq \mathrm{P}_{\mathcal{D}}$ $\Longleftrightarrow \mathrm{OptP} \subseteq \mathrm{P}_{\mathcal{D}}$.

Proof. $(\Longleftarrow)$ Let $L$ be any set in $\Delta_{2}^{\mathrm{P}}$. By using the proof technique for [Kre88, Theorem 3.1], we can define some function opt-val in OptP such that $x \in L$ if and only if the last bit of opt-val( $(x)$ is 1 . Thus, for any distribution $\mu \in \mathcal{D}$, if opt-val is polynomial-time computable on $\mu$-average, so is $L$.

$(\Longrightarrow)$ Let opt-val be any function in OptP. We assume that for some polynomial $r$, $|\operatorname{opt} \operatorname{val}(x)| \leq r(|x|)$ for all $x \in \Sigma^{*}$. It is easy to show that the following set $L$ is in $\Delta_{2}^{\mathrm{P}}$ :

$$
\begin{aligned}
L=\left\{\langle n, x, i, d\rangle_{n}:\right. & x \in \Sigma^{n}, 1 \leq i \leq r(n), d \in\{0,1\}, \text { and } \\
& \text { the } i \text { th bit of the lexicographically smallest opt-vall(x) is } d\} .
\end{aligned}
$$

Clearly, for any $x \in \Sigma^{*}$, some value of opt-val( $x$ ) (in fact, the lexicographically smallest value of opt-val( $(x))$ is polynomial-time computable by asking at most $q(|x|)$ queries to $L$. Then it is easy to show that $(o p t-v a l, \mu)$ is $\alpha_{\mathrm{T}}^{\mathrm{P}}$-reducible (in fact, $\alpha_{\mathrm{tt}}^{\mathrm{P}}$-reducible) to $\left(L, \mu^{\prime}\right)$ for some $\mu^{\prime} \in \mathrm{P}_{\mathcal{D}}$. Then the proof follows from Proposition 2.8.

Now we are ready to prove our first main result. That is, $\mathrm{NP} \subseteq \mathrm{P}_{\mathrm{P}^{\mathrm{NP}} \text {-samp }}$ is sufficient and indeed necessary for showing $\Delta_{2}^{\mathrm{P}} \subseteq \mathrm{P}_{\mathrm{P} \text {-comp }}$ (i.e., $\mathrm{OptP} \subseteq \mathrm{P}_{\mathrm{P} \text {-comp }}$ ). First we show the sufficiency. In fact, we can prove that $\mathrm{NP} \subseteq \mathrm{P}_{\mathrm{P}^{N P} \text {-samp }}$ is sufficient for $\Delta_{2}^{\mathrm{P}} \subseteq \mathrm{P}_{\mathrm{P}^{N P} \text {-samp. }}$.

Theorem 4.2. If $\mathrm{NP} \subseteq \mathrm{P}_{\mathrm{P}^{N P} \text {-samp }}$, then $\Delta_{2}^{\mathrm{P}} \subseteq \mathrm{P}_{\mathrm{P}^{N P} \text {-samp }}$.

Proof. Let $L_{1}$ be any set in $\Delta_{2}^{\mathrm{P}}$ and $\mu_{1}$ be any $\mathrm{P}^{\mathrm{NP}}$-samplable distribution. Then we have a polynomial-time bounded deterministic Turing machine $Q$ and an NP set $L_{2}$ such that $Q^{L_{2}}$ accepts $L_{1}$. Also there exist a polynomial-time bounded randomized Turing 
machine $G$ and an NP set $X$ witnessing $\mu_{1}$ is $\mathrm{P}^{\mathrm{NP}}$-samplable. We may assume, for some polynomial $q_{1}$ and all $x \in \Sigma^{n}$, that $Q^{L_{2}}(x)$ asks exactly $q_{1}(n)$ distinct queries, and that it always asks queries of the form $\langle n, x, y\rangle_{n}$ for some $y$.

Now for any $\langle n, x, y\rangle_{n}$ that is queried by $Q^{L_{2}}(x)$, define $\mu_{2}\left(\langle n, x, y\rangle_{n}\right)=\mu_{1}(x) / q_{1}(n)$, and $\mu_{2}(z)=0$ for any other $z$. Then $\mu_{2}$ is a well-defined probability function. Also it is clear that $\mu_{2}$ satisfies the condition (c) of Definition 2.7 for $L_{1}, L_{2}, Q$, and $\mu_{1}$ by taking $p(n)=1$ and $q(n)=q_{1}(n)$. Hence, $\left(L_{1}, \mu_{1}\right)$ is $\propto_{\mathrm{T}}^{\mathrm{P}}$-reducible to $\left(L_{2}, \mu_{2}\right)$.

On the other hand, for a given $n \geq 0$, one can generate each $\langle n, x, y\rangle_{n}$ with probability $\mu_{2}\left(\langle n, x, y\rangle_{n}\right)$ in the following way: First, simulate $G^{X}(\bar{n})$ to generate $x$. Then simulate $Q^{L_{2}}(x)$ to generate all queries. Finally, output one of the generated queries at random. Thus, $\mu_{2}$ is $\mathrm{P}^{X \oplus L_{2}}$-samplable. Therefore, by assumption, $L_{2}$ is polynomial-time solvable on $\mu_{2}$-average, which proves that $L_{1}$ is polynomial-time solvable on $\mu_{1}$-average because $\left(L_{1}, \mu_{1}\right) \propto_{\mathrm{T}}^{\mathrm{P}}\left(L_{2}, \mu_{2}\right)$.

Next we show that $\mathrm{NP} \subseteq \mathrm{P}_{\mathrm{P}^{\mathrm{NP}} \text {-samp }}$ is indeed necessary for showing $\Delta_{2}^{\mathrm{P}} \subseteq \mathrm{P}_{\mathrm{P} \text {-comp }}$. That is, the following relation.

Theorem 4.3. If $\Delta_{2}^{\mathrm{P}} \subseteq \mathrm{P}_{\mathrm{P}-\text { comp }}$, then $\mathrm{NP} \subseteq \mathrm{P}_{\mathrm{P}_{\mathrm{NP}} \text {-samp }}$.

Suppose $\Delta_{2}^{\mathrm{P}} \subseteq \mathrm{P}_{\mathrm{P}-\text { comp }}$, and let us first discuss very intuitively why this seems to

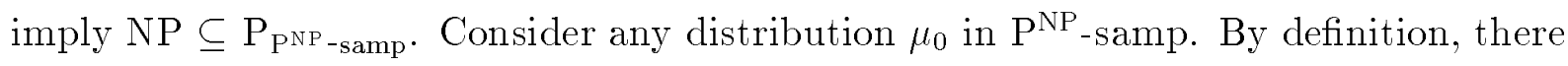
exist a randomized machine $G_{0}$ and a NP oracle set $X_{0}$ such that $G_{0}^{X_{0}}(\bar{n})$ produces $x \in \Sigma^{n}$ according to $\mu_{0}$. Since $\Delta_{2}^{\mathrm{P}} \subseteq \mathrm{P}_{\mathrm{P} \text {-comp }}$, every $\mathrm{P}^{\mathrm{NP}}$-computation can be simulated by some randomized machine whose running time is polynomial on average; thus, the computation of $G_{0}^{X_{0}}$ can be simulated by some randomized machine $G_{0}^{\prime}$ whose running time is polynomial on average. That is, the distribution $\mu_{0}$ itself is "on average" Psamplable, or, one can define a P-samplable distribution $\mu_{0}^{\prime}$ that "approximates" $\mu_{0}$. Since $\Delta_{2}^{\mathrm{P}} \subseteq \mathrm{P}_{\mathrm{P}-\text { comp }}$, clearly $\mathrm{NP} \subseteq \mathrm{P}_{\mathrm{P}-\text { comp }}$. Thus, by Proposition 3.3, $\mathrm{NP} \subseteq \mathrm{P}_{\mathrm{P} \text {-samp }}$; that is, NP sets are decidable in polynomial-time on $\mu$-average for any $\mathrm{P}$-samplable distribution $\mu$. Hence, NP sets are decidable in polynomial-time on $\mu_{0}^{\prime}$-average. But this property seems to hold for $\mu_{0}$ because $\mu_{0}^{\prime}$ is a "good approximation" of $\mu_{0}$.

This intuitive idea can be formalized to prove a similar relation for weaker "average polynomial-time" notions such as our almost polynomial-time criteria (Definition 2.6). On the other hand, for proving the above theorem, we need a more careful argument. We begin by defining the notion of "polynomial-time samplable on average".

For any probability function $\mu$, we say that $\mu$ is aveP-samplable distribution if there exists a deterministic Turing machine $G$ such that for each $n \geq 0$, 
(1) for every $s, s^{\prime} \in \Sigma^{*}$, if $G(\bar{n}, s) \neq \perp$, then $G\left(\bar{n}, s s^{\prime}\right)=\perp$.

(2) for every $x \in \Sigma^{n}, \mu(x)=\lambda\left\{s \in \Sigma^{*}: G(\bar{n}, s)\right.$ yields $\left.x\right\}$, and

(3) $G$ runs polynomial-time on $\lambda$-average; that is, there exist constants $c, d>0$ (independent from $n$ ) such that

$$
\sum_{s \in \Sigma^{*} \wedge G(\bar{n}, s) \neq \perp} \frac{\left(\operatorname{time}_{G}(\bar{n}, s)\right)^{1 / d}}{n} \lambda(s) \leq c .
$$

Here we use $\lambda$ to denote the uniform distribution on $\Sigma^{*}$. That is, $G(\bar{n}, s)$ (with $G(\bar{n}, s) \neq \perp)$ is regarded as the execution of randomized generator that halts after consuming all bits of $s$ for its coin-tossing. In the following, we will refer $G$ satisfying (1) and (2) as a deterministic generator for $\mu$. Notice that the generator $G$ in Definition 2.2 can be modified to this type of generator that halts always in polynomial-time. Thus, this notion is a natural generalization of $\mathrm{P}$-samplable distribution. We use aveP-samp to denote the class of aveP-samplable distributions.

Now we prove the following theorem, which is a generalization of Proposition 3.3.

Theorem 4.4. $\mathrm{NP} \subseteq \mathrm{P}_{\mathrm{P}-\text { comp }} \Longrightarrow \mathrm{NP} \subseteq \mathrm{P}_{\text {aveP-samp }}$.

Proof. Consider any set $L_{0}$ in NP and any aveP-samplable distribution $\mu_{0}$. We will construct some machine $M_{2}$ that recognizes $L_{0}$ in polynomial-time on $\mu_{0}$-average.

Let $G_{0}$ be a deterministic generator for $\mu_{0}$ that satisfies the condition $(1) \sim(3)$ above. We consider any $n \geq 0$; let us fix it for a while, and discuss only inputs in $\Sigma^{n}$. For simplifying notation, in the following, we write $G(\bar{n}, s)$ as $G(s)$.

Note that $L_{0} \in \mathrm{NP}$ is solvable in exponential-time. Hence, for some polynomial $e_{0}$, we may consider only instances $x$ with $\mu(x) \geq 2^{-\epsilon_{0}(n)+1}$; that is, we can use the exponential-time algorithm for $L_{0}$ for those instances $x$ with $\mu(x)<2^{-\epsilon_{0}(n)+1}$, and this does not affect the polynomial-time computability discussion. Thus, in the following, we may assume that $\mu_{0}(x) \geq 2^{-e_{0}+1}$. (Again we use $e_{0}$ to denote $e_{0}(n)$.) On the other hand, for each $x \in \Sigma^{n}$, we have $\lambda\left\{s: G_{0}(s)=x \wedge G_{0}(s)\right.$ takes more than $\left(2^{e_{0}} c_{0} n\right)^{d_{0}}$ steps $\} \leq 2^{-e_{0}}$. Thus, even if we modify $G_{0}$ so that it terminates after $\left(2^{e_{0}} c_{0} n\right)^{d_{0}}$ steps, the probability that $x$ is generated is reduced by only $2^{-e_{0}}$, which is at most the half of $\mu_{0}(x)$, since we have just assumed that $\mu_{0}(x) \geq 2^{-\epsilon_{0}+1}$. Hence, without losing generality, we may assume that the worst-case running time of $G_{0}(s)$ is bounded by $\left(2^{e_{0}} c_{0} n\right)^{d_{0}}$.

From the condition (2), $G_{0}$ runs in polynomial-time on $\lambda$-average, which is witnessed by constants $c_{0}, d_{0}>0$. This intuitively means that the average running time of $G_{0}(s)$ is $\left(c_{0} n\right)^{d_{0}}$. That is, with $t(u)=\left(2^{u} G_{0} n\right)^{d_{0}}$, On the other hand, as explained above, the worst-case running time of $G_{0}(s)$ is bounded by $\left(2^{e_{0}} c_{0} n\right)^{d_{0}}$. Hence, with $t(u)=$ 
$\left(2^{u} G_{0} n\right)^{d_{0}}$, we can claim that the running time of $G_{0}$ for producing some string of length $n$ is bounded by $t(0)$ on average, and bounded by $t\left(e_{0}\right)$ in the worst case. Thus, intuitively, for most of $s \in \Sigma^{*}$ such that $G_{0}(s) \neq \perp, G_{0}(s)$ halts in $t(0)$ steps, and the number of $s$ for which $G_{0}(s)$ needs more than $t(u)$ steps becomes less and less when $u$ increases.

For any $u, 0 \leq u \leq e_{0}$, define $G_{0}^{(u)}, \mu_{0}^{(u)}$, and $\widehat{\mu_{0}}$ as follows:

$$
\begin{aligned}
& G_{0}^{(u)}(s)=\left\{\begin{array}{cc}
G_{0}(s), & \text { if }\left(2^{u-1} c_{0} n\right)^{d_{0}}<\operatorname{time}_{G_{0}}(s) \leq\left(2^{u} c_{0} n\right)^{d_{0}}, \text { and } \\
\perp, & \text { otherwise. }
\end{array}\right. \\
& \text { (For } u=0 \text { use the condition time } G_{0}(s) \leq\left(c_{0} n\right)^{d_{0}} \text { instead.) } \\
& \mu_{0}^{(u)}(x)=\lambda\left(\left(G_{0}^{(u)}\right)^{-1}(x)\right) . \quad \widehat{\mu}_{0}(u)=\sum_{x \in \Sigma^{n}} \mu_{0}^{(u)}(x) .
\end{aligned}
$$

Then the following relations are clear from the definition.

\section{Fact 1.}

(1) For any $x \in \Sigma^{n}$, we have $\mu_{0}(x)=\sum_{u=0}^{e_{0}} \mu_{0}^{(u)}(x)$. Hence, $\sum_{u=0}^{e_{0}} \widehat{\mu_{0}}(u)=1$.

(2) For any $u, 0 \leq u \leq e_{0}$, we have $\widehat{\mu_{0}}(u) \times 2^{u-1} \leq 1$.

It follows from the above (1) that for any $x$, there exists some $u, 0 \leq u \leq e_{0}$, such that $\mu_{0}^{(u)}(x) \geq \mu_{0}(x) /\left(e_{0}+1\right)$. Let $u_{x}$ denote it. Intuitively, for most of random seeds generating $x, G_{0}$ runs about $t\left(u_{x}\right)$ steps.

Here for the proof, we make use of one key lemma, which is provable by a straightforward modification of [IL90]. Let us first review [IL90]. Consider any set $L$ in NP and any $\mathrm{P}$-samplable distribution $\mu$. Hence, $\mu$ has a polynomial-time bounded generator $G$. From the assumption that $\mathrm{NP} \subseteq \mathrm{P}_{\mathrm{P}-\text { comp }}$, Impagliazzo and Levin constructed some randomized machine $M$ such that (1) $M$ recognizes $L$, and (2) $M$ runs in polynomialtime on $\mu$-average. By modifying their argument (see Appendix for the detail), we can construct the following $M_{1}$ for our $L_{0}$ and $G_{0}$.

Lemma 4.5. There exists a randomized machine $M_{1}$ with the following properties:

(1) For all $x \in \Sigma^{n}$ and $u \geq 0$,

(i) $M_{1}\left(\langle n, \overline{t(u)}, x\rangle_{n, t(u)}\right)$ outputs either 0 , 1 , or $\perp$,

(ii) if $M_{1}\left(\langle n, \overline{t(u)}, x\rangle_{n, t(u)}\right) \neq \perp$, then $M_{1}\left(\langle n, \overline{t(u)}, x\rangle_{n, t(u)}\right)=1 \Longleftrightarrow x \in L_{0}$, and

(iii) if $\mu_{0}^{(u)}(x) \neq 0$, then $\operatorname{Pr}_{M_{1}}\left\{M_{1}\left(\langle n, \overline{t(u)}, x\rangle_{n, t(u)}\right) \neq \perp\right\} \geq 2 / 3$.

(2) $M_{1}$ runs in polynomial time on $\mu_{1}$-average, where $\mu_{1}\left(\langle n, \overline{t(u)}, x\rangle_{n, t(u)}\right)=\mu_{0}^{(u)}(x)$ for any $u, 0 \leq u \leq e_{0}$. (We artificially set $\mu_{1}\left(\langle n, 0,0\rangle_{n, t(u)}\right)=1-\widehat{\mu_{0}}(u)$, and $\mu_{1}\left(x^{\prime}\right)=0$ for any other form of $x^{\prime}$.) 
Now by using $M_{1}$, we define the following algorithm $M_{2}$.

$\operatorname{prog} M_{2}($ input $x)$

$n \leftarrow|x| ; N \leftarrow p_{1}(n) ; \quad \% p_{1}$ is sufficiently large polynomial.

\section{in parallel do}

(0) simulate $N$ executions of $M_{1}\left(\langle n, \overline{t(0)}, x\rangle_{n, t(0)}\right)$ in parallel by using $N$ randomly chosen sequences as $M_{1}$ 's random resource;

if $M_{1}$ yields $0 / 1$ then accept/reject;

$\left(e_{0}\right)$ simulate $N$ executions of $M_{1}\left(\left\langle n, \overline{t\left(e_{0}\right)}, x\right\rangle_{n, t\left(e_{0}\right)}\right)$ in parallel by using $N$ randomly chosen sequences as $M_{1}$ 's random resource; if $M_{1}$ yields $0 / 1$ then accept/reject;

(*) determine $x \in L_{0}$ by brute force deterministic computation; if the computation accepts/rejects then accept/reject

od.

Clearly, this $M_{2}$ recognizes $L_{0}$ correctly. Thus, it suffices to show that $M_{2}$ runs in polynomial-time on $\mu_{0}$-average. In the following discussion, we consider any sufficiently large $n$, and let it be fixed.

Let $c_{1}$ and $d_{1}$ are constants witnessing that $M_{1}$ is polynomial-time on $\mu_{1}$-average. Thus, for any $u, 0 \leq u \leq e_{0}$,

$$
\sum_{x \in \Sigma^{n}} \frac{\left(t i m e_{M_{1}}\left(\langle n, \overline{t(u)}, x\rangle_{n, t(u)}\right)\right)^{1 / d_{1}}}{\left|\langle n, \overline{t(u)}, x\rangle_{n, t(u)}\right|} \mu_{1}\left(\langle n, \overline{t(u)}, x\rangle_{n, t(u)}\right) \leq c_{1} .
$$

Recall that $\mu_{1}\left(\langle n, \overline{t(u)}, x\rangle_{n, t(u)}\right)=\mu_{0}^{(u)}(x)$ and that $\left|\langle n, \overline{t(u)}, x\rangle_{n, t(u)}\right|$ is less than some polynomial in $n+t(u)$, which is bounded by $\left(2^{u-1} n\right)^{d_{2}}$ for some $d_{2}$. Thus, for any $u$,

$$
\sum_{x \in \Sigma^{n}} \frac{\left(t i m e_{M_{1}}\left(\langle n, \overline{t(u)}, x\rangle_{n, t(u)}\right)\right)^{1 / d_{1}}}{\left(2^{u-1} n\right)^{d_{2}}} \mu_{0}^{(u)}(x) \leq c_{1} .
$$

Note also that $\sum_{x \in \Sigma^{n}} \mu_{0}^{(u)}(x)=\widehat{\mu_{0}}(u) \leq 1 / 2^{u-1}$ for any $u$. Thus, for any $u, 0 \leq u \leq e_{0}$, it follows from Proposition 2.5 that

$$
\sum_{x \in \Sigma^{n}} \frac{\left(t i m e_{M_{1}}\left(\langle n, \overline{t(u)}, x\rangle_{n, t(u)}\right)\right)^{1 /\left(2 d_{1} d_{2}\right)}}{n^{d_{2}}} \mu_{0}^{(u)}(x) \leq c_{1}+1 .
$$

Now for a given input $x \in \Sigma^{n}$, estimate the running time of $M_{2}(x)$. Here we focus on the $u_{x}$ th parallel step; that is, the simulation of $M_{1}\left(\left\langle n, \overline{t\left(u_{x}\right)}, x\right\rangle_{n, t\left(u_{x}\right)}\right)$. Recall that the probability that $M_{1}\left(\left\langle n, \overline{t\left(u_{x}\right)}, x\right\rangle_{n, t\left(u_{x}\right)}\right) \neq \perp$ is greater than $2 / 3$, and 
that $M_{1}\left(\left\langle n, \overline{t\left(u_{x}\right)}, x\right\rangle_{n, t\left(u_{x}\right)}\right)$ is simulated by using $N$ random sequences in parallel. Thus, with very high probability, some simulation of $M_{1}\left(\left\langle n, \overline{t\left(u_{x}\right)}, x\right\rangle_{n, t\left(u_{x}\right)}\right)$ yields 0 or 1 , and furthermore it halts in $2 \cdot$ time $_{M_{1}}\left(\left\langle n, \overline{t\left(u_{x}\right)}, x\right\rangle_{n, t\left(u_{x}\right)}\right)$ steps. (Recall that time $_{M_{1}}\left(\left\langle n, \overline{t\left(u_{x}\right)}, x\right\rangle_{n, t\left(u_{x}\right)}\right)$ is the expected running time over all random sequences of $\left.M_{1}\right)$. Thus, we can assume that the running time of $M_{2}(x)$ is bounded by that of the $u_{x}$ th parallel step, which is bounded by $2 N$ time $_{M_{1}}\left(\left\langle n, \overline{t\left(u_{x}\right)}, x\right\rangle_{n, t\left(u_{x}\right)}\right)+p_{2}\left(2^{u_{x}} n\right)$ for some polynomial $p_{2}$.

Hence, for some constant $d_{3} \geq 2 d_{1} d_{2}$ such that $\left(p_{2}\left(2^{u_{x}} n\right)\right)^{1 / d_{3}} \leq 2^{u_{x}-1} n$ we have

$$
\begin{aligned}
& \sum_{x: u_{x}=u} \frac{\left(\operatorname{time}_{M_{2}}(x)\right)^{1 / d_{3}}}{n} \mu_{0}(x) \\
& \leq \quad\left(e_{0}+1\right) \cdot \sum_{x: u_{x}=u} \frac{\left(2 N \operatorname{time}_{M_{1}}\left(\left\langle n, \overline{t\left(u_{x}\right)}, x\right\rangle_{n, t\left(u_{x}\right)}\right)+p_{2}\left(2^{u_{x}} n\right)\right)^{1 / d_{3}}}{n} \mu_{0}^{\left(u_{x}\right)}(x) \\
& \leq \quad\left(e_{0}+1\right) n^{d_{2}-1} \cdot \sum_{x: u_{x}=u} \frac{\left(2 N \operatorname{time}_{M_{1}}\left(\left\langle n, \overline{t\left(u_{x}\right)}, x\right\rangle_{n, t\left(u_{x}\right)}\right)\right)^{1 / d_{3}}}{n^{d_{2}}} \mu_{0}^{\left(u_{x}\right)}(x) \\
& \quad+\left(e_{0}+1\right) \cdot \sum_{x: u_{x}=u} \frac{\left(p_{2}\left(2^{u_{x}} n\right)\right)^{1 / d_{3}}}{n} \mu_{0}^{\left(u_{x}\right)}(x) \\
& \leq \quad\left(e_{0}+1\right) n^{d_{2}-1}(2 N)^{1 / d_{3}}\left(c_{1}+1\right)+\left(e_{0}+1\right) \cdot \frac{2^{u_{x}-1} n}{n} \cdot \frac{1}{2^{u_{x}-1}} \\
& \leq \quad\left(e_{0}+1\right)\left(n^{d_{2}-1}(2 N)^{1 / d_{3}}\left(c_{1}+1\right)+1\right) \cdot
\end{aligned}
$$

Hence, summing up this for $u=0$ to $u=e_{0}(n)$, we have $\sum_{x \in \Sigma^{n}}\left(\operatorname{time}_{M_{1}}(x)\right)^{1 / d_{2}} / n \leq$ $p_{3}(n)$ for some polynomial $p_{3}$. Therefore, from Proposition 2.4, we conclude that $M_{2}$ runs in polynomial-time on $\mu_{1}$-average.

Now with Theorem 4.4, the proof of Theorem 4.3 is easy.

Proof of Theorem 4.3. It suffices to show that under the assumption that $\Delta_{2}^{\mathrm{P}}$ $\subseteq \mathrm{P}_{\mathrm{P} \text {-comp }}$, every $\mathrm{P}^{\mathrm{NP}}$-samplable distribution is aveP-samplable. Consider any $\mathrm{P}^{\mathrm{NP}}$ samplable distribution $\mu$. Then there exists some $\mathrm{P}^{\mathrm{NP}}$-generator $G^{X}$ for $\mu$. That is, $G$ is a polynomial-time bounded deterministic machine, $X$ is a set in NP, and they satisfies the following for some polynomial $r$ and all $n \geq 0$ and $x \in \Sigma^{n}$ :

$$
\mu(x)=\lambda\left\{s \in \Sigma^{r(n)}: G^{X}(\bar{n}, s)=x\right\}
$$

Then define $L$ by

$$
L=\left\{\langle n, s, i, d\rangle_{n}: n \geq 0, s \in \Sigma^{r(n)}, 1 \leq i \leq n, d \in\{0,1\} \text {, and }\left(G^{X}(\bar{n}, s)\right)_{i}=d\right\}
$$


where $\left(G^{X}(\bar{n}, s)\right)_{i}$ is the $i$ th bit of $G^{X}(\bar{n}, s)$. Clearly, $L$ is in $\Delta_{2}^{\mathrm{P}}$. Thus, it is polynomialtime decidable on average under uniform distribution. On the other hand, the function $G^{X}$ (under uniform distribution) is $\propto_{\mathrm{tt}}^{\mathrm{P}}$-reducible to $L$ (under uniform distribution). Therefore, $G^{X}$ is computed by some $G^{\prime}$ that runs in polynomial-time on average under uniform distribution.

\section{The Second Question}

In this section, we discuss the second question. That is, from the assumption NP $\subseteq$ $\mathrm{P}_{\mathrm{P}-\text { comp }}$, how far can we prove? Or more specifically, for which distribution class $\mathcal{D}$, can we prove $\mathrm{NP} \subseteq \mathrm{P}_{\mathcal{D}}$ ?

We first show that the assumption implies $\mathrm{NP} \subseteq \mathrm{P}_{\mathrm{P}_{\mathbf{t t}}^{\mathrm{NP}} \text {-samp. }}$.

Theorem 5.1. If $\mathrm{NP} \subseteq \mathrm{P}_{\mathrm{P}-\text { comp }}$, then $\mathrm{NP} \subseteq \mathrm{P}_{\mathrm{P}_{\mathrm{tt}}^{\mathrm{NP}}-\mathrm{samp}}$.

Proof. The proof is essentially the same as the one for Theorem 4.3. It follows from Proposition 3.3 that the assumption implies that $\mathrm{P}_{\mathrm{tt}}^{\mathrm{NP}} \subseteq \mathrm{P}_{\mathrm{P}-\text { comp }}$. Then by exactly the same argument as the one for Theorem 4.3, we can show, for every $\mathrm{P}_{\mathrm{tt}}^{\mathrm{NP}}$-samplable distribution $\mu$, that $\mu$ is aveP-samplable. Thus, by Theorem 4.4, every NP set is polynomial-time solvable on $\mu$-average. That is, $\mathrm{NP} \subseteq \mathrm{P}_{\mathrm{P}_{\mathrm{tt}}^{\mathrm{NP}} \text {-samp }}$.

Therefore, the difference between $\mathrm{P}_{\mathrm{P}_{\mathrm{tt}}^{\mathrm{NP}} \text {-samp }}$ and $\mathrm{P}_{\mathrm{PNP}_{\text {-samp }}}$ is essential for our original question, namely, the question of whether it holds that $\mathrm{NP} \subseteq \mathrm{P}_{\mathrm{P} \text {-comp }} \Longrightarrow \mathrm{OptP} \subseteq$ $\mathrm{P}_{\mathrm{P} \text {-comp. }}$ This leads us to another type of question. That is, the relation between $\mathrm{P}_{\mathrm{P}_{\mathrm{tt}}^{N P}-\text { samp }}$ and $\mathrm{P}_{\mathrm{P}^{N P}-\text { samp }}$, or in more general, between distribution classes $\mathrm{P}_{\mathrm{tt}}^{\mathrm{NP}}$-samp and $\mathrm{P}^{\mathrm{NP}}$-samp.

Here we show that the class $\mathrm{P}_{\mathrm{tt}}^{\mathrm{NP}}$-samp is in fact not so small by proving that it (essentially) contains some distribution class, i.e., the class of \#P-computable distributions, which seems much stronger than P-comp. Let us first define the notion of "\#P-computable distribution". Intuitively, an input distribution $\mu$ is a \#P-computable if $\mu$ is defined as some \#P function. That is, there exists a polynomial-time computable binary predicate $R$ and a polynomial $q$ such that for each $x$ (let $n=|x|$ ),

$$
\mu(x)=\frac{\left\|\left\{w \in \Sigma^{q(n)} \mid R(x, w)\right\}\right\|}{2^{q(n)}} .
$$

Let \#P-comp denote the class of \#P-computable distributions.

For any two input distributions $\mu_{1}$ and $\mu_{2}$, we say that $\mu_{1}$ approximates $\mu_{2}$ within constant factor if $c_{1} \mu_{1}(x) \leq \mu_{2}(x) \leq c_{2} \mu_{1}(x)$ for some constants $c_{1}, c_{2}>0$ and for all $x \in \Sigma^{*}$. We have the following theorem. 
Theorem 5.2. Every input distribution in \#P-comp is approximated within constant factor by some input distribution in $\mathrm{P}_{\mathrm{tt}}^{\mathrm{NP}}$-samp.

For the proof we use hashing functions. Here we use linear hashing functions of Carter and Wegman [CW79] for our concrete hashing functions. A linear hash function $h$ from $\Sigma^{l}$ to $\Sigma^{m}$ is given by a Boolean $(m, l)$-matrix $A=\left(a_{i, j}\right)$, and maps any string $x=x_{1} \ldots x_{l} \in \Sigma^{l}$ to some string $y=y_{1} \ldots y_{m}$, where $y=A x$ under modulo 2. Let $H_{l, m}$ be the set of linear hash functions from $\Sigma^{l}$ to $\Sigma^{m}$. The following facts are basic properties of linear hash functions.

Fact 2. For all $x \in \Sigma^{l}$ and for all $y \in \Sigma^{m}$,

$$
\operatorname{Pr}_{h \in H_{l, m}}\{h(x)=y\}=\frac{1}{2^{m}} .
$$

Fact 3. For all $x_{1}, x_{2} \in \Sigma^{l}, x_{1} \neq x_{2}$, and for all $y_{1}, y_{2} \in \Sigma^{m}$,

$$
\begin{aligned}
& \operatorname{Pr}_{h \in H_{l, m}}\left\{h\left(x_{2}\right)=y_{2} \mid h\left(x_{1}\right)=y_{1}\right\}=\frac{1}{2^{m}}, \text { and thus } \\
& \operatorname{Pr}_{h \in H_{l, m}}\left\{h\left(x_{1}\right)=y_{1} \wedge h\left(x_{2}\right)=y_{2}\right\}=\frac{1}{2^{2 m}} .
\end{aligned}
$$

We make use of the following lemma.

Lemma 5.3. Let $X$ be any subset of $\Sigma^{l}$ of size $K=2^{k}$ for some $k \geq 0$, and let $m=k+c$ for some integer $c>0$. For all $x \in X$ define $P_{x}$ by

$$
P_{x}=\operatorname{Pr}\left\{(h(x)=y) \wedge\left(\forall x^{\prime}: x^{\prime} \in X \wedge x^{\prime} \neq x\left[h\left(x^{\prime}\right) \neq y\right]\right) \mid h \in H_{l, m}, y \in \Sigma^{m}\right\} .
$$

Then $P_{x}$ is bounded as follows:

$$
\left(1-\frac{1}{2^{c}}\right) \cdot \frac{1}{2^{c}} \times \frac{1}{K} \leq P_{x} \leq \frac{1}{K}
$$

Proof. In the following, we assume that hashing function $h$ is chosen from $H_{l, m}$ randomly and that the domain of $y$ is $\Sigma^{m}$. First we have

$$
\begin{aligned}
P_{x} & =\sum_{y} 2^{-m} \cdot \operatorname{Pr}\left\{\forall x^{\prime}: x^{\prime} \in X \wedge x^{\prime} \neq x\left[h\left(x^{\prime}\right) \neq y\right] \mid h(x)=y\right\} \cdot \operatorname{Pr}\{h(x)=y\} \\
& =\sum_{y} 2^{-m} \cdot 2^{-m}\left(1-\operatorname{Pr}\left\{\exists x^{\prime} \in X\left[x^{\prime} \neq x \wedge h\left(x^{\prime}\right)=y\right] \mid h(x)=y\right\}\right) \\
& \geq \sum_{y} 2^{-m}\left(1-\frac{\|X\|-1}{2^{k+c}}\right) \times \frac{1}{2^{k+c}} \geq\left(1-\frac{1}{2^{c}}\right) \frac{1}{2^{c}} \times \frac{1}{K} .
\end{aligned}
$$

On the other hand, we have 


$$
P_{x} \leq \operatorname{Pr}\left\{h(y)=x \mid y \in \Sigma^{m}, h \in H_{l, m}\right\} \leq \sum_{y} 2^{-m} \cdot 2^{-m}=2^{-m} \leq \frac{1}{K} .
$$

Jerrum, Valiant, and Vazirani [JVV86] showed a method to generate, for any set $X$ in $\mathrm{P}$ and a given $l$, a string of length $l$ in $X$ with almost the same probability in polynomial-time by using some NP oracle. The above lemma gives a much simpler method to do the same task provided we know the size of $X \cap \Sigma^{l}$; in fact, the method uses an NP oracle only nonadaptively. This point is crucial for proving our theorem.

Proof of Theorem 5.2. Let $\mu$ be any input distribution in \#P-comp. Then by definition, there exists a polynomial $q$ and a polynomial-time computable binary predicate $R$ such that for any $n \geq 0$ and any $x \in \Sigma^{n}, \mu(x)=\left\|\left\{w \in \Sigma^{q(n)} \mid R(x, w)\right\}\right\| / 2^{q(n)}$. We first define the following sets $X_{1}$ and $X_{2}$ :

$$
\begin{aligned}
& X_{1}=\{\{n, h, y\rangle: n \geq 0, h \in H_{n+q(n), q(n)+1}, y \in \Sigma^{q(n)+1}, \text { and } \\
& \exists x, w, x^{\prime}, w^{\prime}: x, x^{\prime} \in \Sigma^{n} \wedge w, w^{\prime} \in \Sigma^{q(n)} \\
& {\left.\left[x w \neq x^{\prime} w^{\prime} \wedge h(x w)=h\left(x^{\prime} w^{\prime}\right)=y \wedge R(x, w) \wedge R\left(x^{\prime}, w^{\prime}\right)\right]\right\} . } \\
& X_{2}=\{\langle n, h, y, i, d\rangle: \\
& n \geq 0, h \in H_{n+q(n), q(n)+1}, y \in \Sigma^{q(n)+1}, 1 \leq i \leq n+q(n), d \in\{0,1\}, \text { and } \\
&\left.\exists x, w: x \in \Sigma^{n} \wedge w \in \Sigma^{q(n)}\left[h(x w)=y \wedge R(x, w) \wedge(x w)_{i}=d\right]\right\} .
\end{aligned}
$$

(where $(x w)_{i}$ is the $i$ th bit of $w$ )

Next consider the following randomized machine $G$, and for any $x \in \Sigma^{n}$, define $\mu^{\prime}(x)$ to be the probability that $G(\bar{n})$ generates $x$.

\section{prog G (input $\bar{n})$;}

generate $h$ from $H_{n+q(n), q(n)+1}$ randomly;

generate $y \in \Sigma^{q(n)+1}$ randomly;

if $\langle n, h, y\rangle \in X_{1}$ then output ? and halt;

use oracle $X_{2}$ to find some $x w \in \Sigma^{n+q(n)}$ with $h(x w)=y$ and $R(x, w)$;

if no such $x w$ exists then output ? else output $x$.

Then clearly both $X_{1}$ and $X_{2}$ are in NP. Furthermore, it is easy to modify $G$ so that $G$ asks only nonadaptive queries to $X_{1} \oplus X_{2} \in$ NP. Thus, $G$ can be considered as a $\mathrm{P}_{\mathrm{tt}}^{\mathrm{NP}}$-generator. On the other hand, it follows from Lemma 5.3 that $\mu^{\prime}$ approximates $\mu$ within constant factor. This almost proves the theorem.

Here, precisely speaking, $\mu^{\prime}$ is not a real input distribution because $\mu^{\prime}$ may assign some positive value to error symbol '?'. It is possible, however, to modify $G$ to use polynomially many $y$ 's in parallel and thereby reducing the probability to yield '?' to less than $2^{-q(n)}$. Then $G$ can output any string of length $n$ instead of '?' while keeping' 
the property that $\mu^{\prime}$ approximates $\mu$ within constant factor. The detail analysis is left to the reader.

Notice that if $\mu_{1}$ approximates $\mu_{2}$ within constant factor, then the polynomial-time solvability is equivalent between $\mu_{1}$-average and $\mu_{2}$-average. Thus, we have the following corollary.

Corollary 5.4. $\mathrm{P}_{\# \mathrm{P}-\text { comp }} \subseteq \mathrm{P}_{\mathrm{tt}}^{\mathrm{NP}}$-samp.

\section{Acknowledgments}

The second author thanks to Professor Uwe Schöning for inviting him to the Universität Ulm, which made this joint research possible, and to the people in the Abteilung Theoretische Informatik (in particular, Uwe Schöning and Thomas Thierauf) for their warm hospitality to him. The authors have benefitted very much from valuable discussions with Professor Johannes Köbler, Professor Uwe Schöning, and Professor Thomas Thierauf.

\section{References}

[BDG88] J. Balcázar, J. Díaz, and J. Gabarró, Structural Complexity I, EATCS Monographs on Theoretical Computer Science, Springer-Verlag, 1988.

[BDG91] J. Balcázar, J. Díaz, and J. Gabarró, Structural Complexity II, EATCS Monographs on Theoretical Computer Science, Springer-Verlag, 1991.

[BCGL92] S. Ben-David, B. Chor, O. Goldreich, and M. Luby, On the theory of average case complexity, J. Comput. Syst. Sci. 44 (1992), 193-219.

[CW79] J. Carter and M. Wegman, Universal classes of hash functions, J. Comput. Syst. Sci. 18 (1979), 143-154.

[CG93] R. Chang and W. Gasarch, On bounded queries and approximation, in Proc. 34th IEEE Sympos. on Foundations of Computer Science, IEEE (1993), $547-556$.

[Gur91] Y. Gurevich, Average case completeness, J. Comput. Syst. Sci. 42 (1991), $346-398$. 
[IL90] R. Impagliazzo and L. Levin, No better ways to generate hard NP instances than picking uniformly at random, in Proc. 31st IEEE Sympos. on Foundations of Computer Science (1990), 812-821.

[JVV86] M. Jerrum, L. Valiant, and V. Vazirani, Random generation of combinatorial structures from a uniform distribution, Theoret. Comput. Sci. 43 (1986), $169-188$.

[Joh84] D. Johnson, The NP-completeness column: An on going guide, J. Algorithms 5 (1984), 284-299.

[Kre88] M. Krentel, The complexity of optimization problems, J. Comput. Syst. Sci. 36 (1988), 490-509.

[Lev86] L. Levin, Average case complete problems, SIAM J. Comput. 15 (1986), $285-286$.

[SY92] R. Schuler and T. Yamakami, Structural average case complexity, in Proc. 12th Foundations of Software Technology and Theoretical Computer Science, Lecture Notes in Computer Science 652 (1992), 128-139. 


\section{Appendix: Proof of Lemma 4.5}

Let us first review the proof of the following theorem (i.e., Proposition 3.3) proved in [IL90]. Then the lemma is proved by a straightforward modification of their proof.

Theorem A.1. [IL90] $\mathrm{NP} \subseteq \mathrm{P}_{\mathrm{P}-\text { comp }} \Longrightarrow \mathrm{NP} \subseteq \mathrm{P}_{\mathrm{P}-\text { samp }}$.

Suppose that $\mathrm{NP} \subseteq \mathrm{P}_{\mathrm{P} \text {-comp. }}$. Consider any $\mu_{0}$ in $\mathrm{P}_{\mathrm{P}-\text { samp }}$ and any NP set $L_{0}$, and we show that $L_{0}$ is polynomial-time decidable on $\mu_{0}$-average.

We may assume some polynomial-time computable predicate $W_{0}$ and polynomial $p_{0}$ such that for all $x \in \Sigma^{*}$ (letting $\left.n=|x|\right), x \in L_{0} \Longleftrightarrow \exists w \in \Sigma^{p_{0}(n)}\left[W_{0}(x, w)\right]$. Since $\mu_{0}$ is P-samplable, there exist some polynomial-time bounded generator $G_{0}$ for $\mu_{0}$. That is, for some polynomial $r_{0}$ and for all $x \in \Sigma^{n}$,

$$
\mu_{0}(x)=\frac{\left\|\left\{s \in \Sigma^{r_{0}(n)} \mid G_{0}(\bar{n}, s)=x\right\}\right\|}{2^{r_{0}(n)}} .
$$

Now consider any sufficiently large $n$, and let us fix it in the following discussion. For simplifying our notation, we write $G_{0}(\bar{n}, s)$ as $G_{0}(s)$, and $r_{0}(n)$ and $p_{0}(n)$ as $r_{0}$ and $p_{0}$ respectively.

Here again we use linear hash functions. Recall that $H_{l, m}$ is the set of linear hash functions from $\Sigma^{l}$ to $\Sigma^{m}$. For any $x \in \Sigma^{n}, h_{1} \in H_{l_{1}, r_{0}}$, and $h_{2} \in H_{n, l_{2}}$, we say that $\left(h_{1}, h_{2}, z\right)$ determines $x$ if

$$
\begin{aligned}
& \exists v \in \Sigma^{l_{1}}\left[h_{1}(v)=s \wedge f_{0}(s)=x \wedge h_{2}(x)=z\right] \\
& \wedge \forall v^{\prime} \in \Sigma^{l_{1}}\left[\left(h_{1}\left(v^{\prime}\right)=s^{\prime} \wedge f_{0}\left(v^{\prime}\right)=x^{\prime} \neq x\right) \Longrightarrow h_{2}\left(x^{\prime}\right) \neq z\right] .
\end{aligned}
$$

We show that for appropriate choice of $l_{1}$ and $l_{2},\left(h_{1}, h_{2}, h_{2}(x)\right)$ determines $x$ with high probability. In the following, let $k_{x}=\left\lfloor\left\|\log G_{0}^{-1}(x)\right\|\right\rfloor$. Note that $2^{k_{x}} \leq\left\|G_{0}^{-1}(x)\right\|<$ $2^{k_{x}+1}$; hence, $2^{-\left(r_{0}-k_{x}\right)} \leq \mu_{0}(x)<2^{-\left(r_{0}-k_{x}\right)+1}$.

Lemma A.2. For any $x \in \Sigma^{n}$, let $l_{1}=r_{0}-k_{x}-1$ and $l_{2}=l_{1}+2$. Then we have

$$
P_{0}=\operatorname{Pr}\left\{\left(h_{1}, h_{2}, h_{2}(x)\right) \text { determines } x \mid h_{1} \in H_{l_{1}, r_{0}}, h_{2} \in H_{n, l_{2}}\right\} \geq \frac{1}{16} \text {. }
$$

Proof. Let us fix our $x$, and let $z=h_{2}(x)$. In the following, we assume that $h_{1}$ (resp., $h_{2}$ ) is chosen from $H_{l_{1}, r_{0}}$ (resp., $H_{n, l_{2}}$ ) uniformly at random. Define $P_{1}$ and $P_{2}$ as follows $\left(P_{2}\right.$ varies depending on $\left.h_{1}\right)$ :

$$
\begin{gathered}
P_{1}=\operatorname{Pr}_{h_{1}}\left\{\exists v \in \Sigma^{l_{1}}\left[G_{0}\left(h_{1}(v)\right)=x\right]\right\}, \text { and } \\
P_{2}=\operatorname{Pr}_{h_{2}}\left\{\exists v_{1}, v_{2} \in \Sigma^{l_{1}}\left[G_{0}\left(h_{1}\left(v_{1}\right)\right) \neq G_{0}\left(h_{1}\left(v_{2}\right)\right) \wedge\right.\right. \\
\left.\left.h_{2}\left(G_{0}\left(h_{1}\left(v_{1}\right)\right)\right)=h_{2}\left(G_{0}\left(h_{1}\left(v_{2}\right)\right)\right)=z\right]\right\} .
\end{gathered}
$$


Then clearly, $P_{0} \geq P_{1}-\max _{h_{1}} P_{2}$.

First from the following inequalities, we show that $P_{1} \geq 1 / 4$. (In the following, the range of $s$ 's and $v$ 's are $G_{0}^{-1}(x)$ and $\Sigma^{l_{1}}$ respectively.)

$$
\begin{aligned}
P_{1} & \geq \sum_{(s, v)} \operatorname{Pr}_{h_{1}}\left\{h_{1}(v)=s\right\}-\sum_{(s, v),\left(s^{\prime}, v^{\prime}\right)} \operatorname{Pr}_{h_{1}}\left\{h_{1}(v)=s \wedge h_{1}\left(v^{\prime}\right)=s^{\prime}\right\} \\
& =\frac{\overbrace{\left\|G_{0}^{-1}(x)\right\| \times 2^{l_{1}}}^{\#}}{2^{r_{0}}}-\frac{\#(\#-1)}{2} \times \frac{1}{\left(2^{r_{0}}\right)^{2}} \geq \frac{1}{2}\left(1-\frac{1}{2}\right)=\frac{1}{4} .
\end{aligned}
$$

For estimating $P_{2}$, let $F$ be the range of $G_{0} \circ h_{1}$. Then, for all $h_{1},\|F\| \leq 2^{l_{1}}$ and therefore we have the following.

$$
\begin{aligned}
P_{2} & =\operatorname{Pr}_{h_{2}}\left\{\exists x_{1}, x_{2} \in F\left[x_{1} \neq x_{2} \wedge h_{2}\left(x_{1}\right)=h_{2}\left(x_{2}\right)=z\right]\right\} \\
& \leq \sum_{x_{1} \neq x_{2} \in F} \operatorname{Pr}_{h_{2}}\left\{h_{2}\left(x_{1}\right)=h_{2}\left(x_{2}\right)=z\right\} \\
& \leq\|F\|^{2} /\left(2^{l_{2}}\right)^{2} \leq 2^{2 l_{1}-2 l_{2}}=\frac{1}{16} .
\end{aligned}
$$

Now consider a procedure $Q_{0}$ stated below. The idea of $Q_{0}$ is as follows. For a given $x \in \Sigma^{n}$, let $G_{0}^{-1}(x)$ be the set of strings $y$ such that $G_{0}(y)=x$. Choose $k$ randomly from $\left\{0,1, \ldots, r_{0}\right\}$; then with probability $1 / r_{0}$, we have $k=k_{x}\left(k_{x}=\left\lfloor\log \left\|G_{0}^{-1}(x)\right\|\right\rfloor\right)$. If $k=k_{x}$, then by Lemma A.2, for randomly selected hash functions $h_{1}$ and $h_{2}$, $\left(h_{1}, h_{2}, h_{2}(x)\right)$ determines $x$ with probability $\geq 1 / 16$. That is, we can indirectly specify $x$ by $\left(h_{1}, h_{2}, h_{2}(x)\right)$, and thus, $Q_{0}$ can ask a right query to an oracle $L_{0}^{\prime}$, which is essentially the same as asking $x$ to $L_{0}$.

$\operatorname{prog} Q_{0}$ (input $x \in \Sigma^{n}$ );

choose $k$ randomly from $\left\{0, \ldots, r_{0}\right\}$;

$l_{1} \leftarrow r_{0}-k-2 ; l_{2} \leftarrow l_{1}+2 ;$

choose $h_{1}$ randomly from $H_{l_{1}, r_{0}} ;$ choose $h_{2}$ randomly from $H_{n, l_{2}}$;

$y \leftarrow\left\langle n, k, h_{1}, h_{2}, h_{2}(x)\right\rangle_{n}$, and $y_{i} \leftarrow\left\langle n, k, h_{1}, h_{2}, h_{2}(x), i\right\rangle_{n}$ for each $i, 1 \leq i \leq n$; if $y \in E X_{1} \wedge y \notin E X_{2} \wedge \wedge_{i=1}^{n}\left(x_{i}=1 \Longleftrightarrow y_{i} \in E X_{3}\right)$

then if $y \in L_{0}^{\prime}$ then accept else reject

else output?.

Here oracle sets are defined as follows (here let $l_{1}=r_{0}-k-2, l_{2}=l_{1}+2, x=$ $G_{0}\left(h_{1}(v)\right), x_{1}=G_{0}\left(h_{1}\left(v_{1}\right)\right)$, and $\left.x_{2}=G_{0}\left(h_{1}\left(v_{2}\right)\right)\right)$ :

$$
\begin{aligned}
& E X_{1}=\left\{\left\langle n, k, h_{1}, h_{2}, z\right\rangle_{n}: \exists v \in \Sigma^{l_{1}}\left[h_{2}(x)=z\right]\right\} \\
& E X_{2}=\left\{\left\langle n, k, h_{1}, h_{2}, z\right\rangle_{n}: \exists v_{1}, v_{2} \in \Sigma^{l_{1}}\left[x_{1} \neq x_{2} \wedge h_{2}\left(x_{1}\right)=h\left(x_{2}\right)=z\right]\right\} . \\
& E X_{3}=\left\{\left\langle n, k, h_{1}, h_{2}, z, i\right\rangle_{n}: \exists v \in \Sigma^{l_{1}}\left[h_{2}(x)=z \wedge x_{i}=1\right]\right\} . \\
& L_{0}^{\prime}=\left\{\left\langle n, k, h_{1}, h_{2}, z\right\rangle_{n}: \exists v \in \Sigma^{l_{1}}, w \in \Sigma^{p_{0}}\left[z=h_{2}(x) \wedge W_{0}(x, w)\right]\right\} .
\end{aligned}
$$


Following the above argument, we can easily show that $Q_{0}(x)$ gives a correct answer with probability $\geq 1 / 16 r_{0}$. Note also that when $Q_{0}(x)$ says accept/reject, then the answer is always correct.

From the assumption that $\mathrm{NP} \subseteq \mathrm{P}_{\mathrm{P} \text {-comp }}$, sets $E X_{1}, E X_{2}$, and $L_{0}^{\prime}$ are all solvable by some machines $N_{1}, N_{2}$, and $N_{3}$ respectively in polynomial-time under the following uniform distribution (in the following, we use $H_{l_{1}, r_{0}}$ and $H_{n, l_{2}}$ to denote $\left\|H_{l_{1}, r_{0}}\right\|$ and $\left.\left\|H_{n, l_{2}}\right\|\right)$ :

$$
\nu_{1}\left(\left\langle n, k, h_{1}, h_{2}, z\right\rangle_{n}\right)=\frac{1}{r_{0} \cdot H_{l_{1}, r_{0}} \cdot H_{n, l_{2}} \cdot 2^{l_{2}}} .
$$

Similarly, $E X_{3}$ is solvable by some machine $N_{4}$ in polynomial-time under the following uniform distribution:

$$
\nu_{2}\left(\left\langle n, k, h_{1}, h_{2}, z, i\right\rangle_{n}\right)=\frac{1}{r_{0} \cdot H_{l_{1}, r_{0}} \cdot H_{n, l_{2}} \cdot 2^{l_{2}} \cdot n} .
$$

We estimate the computation time of $Q_{0}$ when using these three machines $N_{1} \sim N_{4}$. (In the following, only $N_{1} \sim N_{3}$ (under distribution $\nu_{1}$ ) are investigated; the analysis for $N_{4}$ (under $\nu_{2}$ ) is almost the same and thus omitted.) Unfortunately, we cannot claim that $Q_{0}$ runs in polynomial-time on $\mu_{0}$-average. The problem is that for wrong choice of $k, h_{1}, h_{2}, Q_{0}(x)$ may ask a query $y$ to $N_{1} \sim N_{3}$ with probability much larger than $\nu_{1}(y)$. It is, however, possible to show that if $\left(h_{1}, h_{2}, h_{2}(x)\right)$ determines $x$, then the probability that $y$ is queried is close to $\nu_{1}(y)$.

Define $\left(\mu_{0} \times \lambda\right)$ to be the probability that a string $y$ is queried by $Q_{0}(x)$ when $x$ is given under distribution $\mu_{0}$. That is,

$$
\left(\mu_{0} \times \lambda\right)\left(\left\langle n, k, h_{1}, h_{2}, z\right\rangle_{n}\right)=\mu_{0}(x) \times \operatorname{Pr}_{Q_{0}}\left\{Q_{0}(x) \text { queries }\left\langle n, k, h_{1}, h_{2}, z\right\rangle_{n}\right\} .
$$

Here suppose that $k$ is chosen correctly; that is, $k=k_{x}$. Then for any $\left(h_{1}, h_{2}, h_{2}(x)\right)$ that determines $x$, we have

$$
\begin{aligned}
\left(\mu_{0} \times \lambda\right)\left(\left\langle n, k, h_{1}, h_{2}, h_{2}(x)\right\rangle_{n}\right) & =\mu_{0}(x) \cdot \frac{1}{H_{l_{1}, r_{0}}} \cdot \frac{1}{H_{n, l_{2}}} \approx 2^{-\left(r_{0}-k\right)} \cdot \frac{1}{H_{l_{1}, r_{0}}} \cdot \frac{1}{H_{n, l_{2}}} \\
& \approx 2^{-l_{2}} \cdot \frac{1}{H_{l_{1}, r_{0}}} \cdot \frac{1}{H_{n, l_{2}}} \approx \frac{1}{r_{0} \cdot H_{l_{1}, r_{0}} \cdot H_{n, l_{2}} \cdot 2^{l_{2}}} \\
& =\nu_{1}\left(\left\langle n, k, h_{1}, h_{2}, h_{2}(x)\right\rangle_{n}\right),
\end{aligned}
$$

where $\approx$ means equal up to a polynomial factor. Thus, for those $\left\langle n, k, h_{1}, h_{2}, h_{2}(x)\right\rangle_{n}$ that determine $x$, machines $N_{1} \sim N_{3}$ (and similarly $N_{4}$ ) return the answer within reasonable amount of time. Or more specifically, if we estimate the time complexity of $Q_{0}^{N_{1}, N_{2}, N_{3}, N_{4}}$ only for the case where $k=k_{x}$ and $\left(h_{1}, h_{2}, h_{2}(x)\right)$ determines $x$, then we can show that the running time is polynomial on $\mu_{0}$-average. 
Now for a sufficiently large polynomial $p$, consider a new procedure $Q_{0}^{\prime}$ that runs $Q_{0}$ for $p(n)$ times in parallel and outputs an answer if one of the executions of $Q_{0}$ returns the answer. Recall that there is not so small chance that $k=k_{x}$ and $\left(h_{1}, h_{2}, h_{2}(x)\right)$ determines $x$. Thus, for any input $x$, the probability that it is determined with the correct $k$ by some execution of $Q_{0}$ is very high. This is enough to prove that $\left(Q_{0}^{\prime}\right)^{N_{1}, N_{2}, N_{3}, N_{4}}$ runs in polynomial-time on $\mu_{0}$-average. Therefore we have Theorem A.1.

Lemma 4.5 is proved almost the same way as above. Here we need to consider a generator $G_{0}$ that may not halt in a fixed polynomial-time. However, by considering time bounded version $G_{0}^{(u)}$ of $G_{0}$, we can easily show that $\left(Q_{0}^{\prime}\right)^{N_{1}, N_{2}, N_{3}, N_{4}}$ indeed satisfies the conditions (1) and (2). 